\title{
Effect of bio-seed priming and nano zinc oxide foliar application on quality and productivity of finger millet + greengram intercropping system
}

\author{
C. Mohanasundar* \\ Department of Agronomy, Tamil Nadu Agricultural University, Coimbatore- 641003 \\ (Tamil Nadu), India \\ K. Ramamoorthy \\ Department of Agronomy, Tamil Nadu Agricultural University, Coimbatore- 641003 \\ (Tamil Nadu), India \\ K. R. Latha \\ Department of Agronomy, Tamil Nadu Agricultural University, Coimbatore- 641003 \\ (Tamil Nadu), India \\ P. Santhy \\ Department of Soil Science and Agricultural Chemistry, Tamil Nadu Agricultural University, \\ Coimbatore- 641003 (Tamil Nadu), India \\ C. N. Chandrasekhar \\ Department of Crop Physiology \& ICAR Nodal Officer, O/o Dean (Agri), Tamil Nadu \\ Agricultural University, Coimbatore- 641003 (Tamil Nadu), India

\section{A. Lakshmanan} \\ Department of Nano Science \& Technology, Tamil Nadu Agricultural University, Coimbatore \\ - 641003 (Tamil Nadu), India \\ ${ }^{*}$ Corresponding author. Email: mohanasundaragri@gmail.com
}

\section{How to Cite}

Mohanasundar, C. et al. (2021). Effect of bio-seed priming and nano zinc oxide foliar application on quality and productivity of finger millet + greengram intercropping system. Journal of Applied and Natural Science, 13(4), 1552 - 1562. https://doi.org/10.31018/ jans.v13i4.3148

\section{Abstract}

A critical stage of the plant's life cycle is germination and insufficient seedling emergence contributes to the lower productivity of finger millet. Priming improves seedling emergence, reduces stand establishment time, and improves seedling germination. There is a need to develop a new technology like Nanotechnology that can precisely detect and deliver the right amount of nutrients or other inputs to safe crops for the environment and maximising productivity. A field experiment was conducted during Kharif season to evaluate the response of effective farming practice for sole finger millet + greengram intercropping system under rainfed conditions to varied levels of bio-seed priming and foliar application of nanoparticles on crop growth and productivity. The results of the experiment revealed that finger millet (Eleusine coracana) intercropped with greengram (Vigna radiata) $(2: 1)$ had a significant level $(\leq 0.05)$ increase in growth and yield parameter of finger millet compared to sole finger millet. Application of Prosopis juliflora leaf extract 1 per cent alone + Foliar ZnO nanoparticle @ 500 ppm showed a significant level $(\leq 0.05)$ increase in growth and yield parameter like grain yield $\left(3238.84 \mathrm{~kg} \mathrm{ha}^{-1}\right)$, finger millet equivalent yield (FMEY) (3483.84 $\left.\mathrm{kg} \mathrm{ha}^{-1}\right)$ and straw yield $\left(7393.83 \mathrm{~kg} \mathrm{ha}^{-1}\right)$ compared to Pogamia pinnata leaf extract $1 \%$ alone + Foliar ZnO nanoparticle @ 500 $\mathrm{ppm}$. The present study mainly focussed on cropping system, bio seed priming, and foliar application of nano zinc oxide utilized during rainfed conditions to increase uniform germination, drought resistance and improve crop yield along with nutrient content inseeds.

Keywords: Finger millet, Intercropping, Nanoparticles, Productivity, Seed priming

\section{INTRODUCTION}

Finger millet (Eleusine coracana L.) is one of the predominant millet crops generally known as ragi belongs to the Poaceae family and is enormously cultivated in India, next to sorghum and pearl millet (Dass et al.,
2013). India is the world's leading producer of finger millet, accounting for approximately 85 percent of worldwide production (Divya et al., 2013; Sakamma et al., 2018). Finger millet contains 9.2 percent protein, 76.3 percent carbohydrate, 2.2 percent minerals, 1.3 percent fat and 3.9 percent ash, and vitamin $A$ and $B$. 
The grains are rich in phosphorus, potassium and amino acid and have the richest source of energy calcium (410 mg/100g grain), which is especially important supplement for growing children and aged people (Tomar et al., 2011). Approximately 1.02 million ha of finger millet are planted in India, with annual production of 1.38 million tonnes and an average yield of 1.4 tonnes per hectare. Finger millet occupies a significant portion of the land in Tamil Nadu, covering 0.61 lakh ha, producing 0.11 million tonnes and yielding 1.9 tons per hectare for marginal farmers (Indiastat, 2017).

As per Manjunath et al. (2018), intercropping is a beneficial crop production technique that aims to maximize productivity and profitability over area and time. Intercropping is a strategy that relies on greater exploitation of sunlight, effective usage of nutrients and water, risk reduction, and increased research of environmental growth parameters (Mobasser et al., 2014; Ajibola and Kolawole, 2019). Other benefits of intercropping include higher profitability and lower fixed costs for land as a benefit of growing a second crop in the same field (Thobatsi, 2009). Intercropping millets with other pulses leads to more efficient use of land and other resources. The system's profitability may be increased by selecting appropriate intercrops that vary in length and development in a range of environments (Sadashiv, 2004).

Crops with different growth characteristics are planted together to complement one another, leading to increased resource use efficiency. Because of their capacity to fix and transmit nitrogen, legumes are essential in intercropping systems involving cereals and millets. Finger millet's initially slow growth phase can be used to generate short-duration pulses. Additionally, intercropping with fast-growing pulses will benefit in controlling weeds (Reddy et al., 2021). Priming enhances germination, speeds up seedling emergence time, and increases stand establishment. The overall goal of seed priming is to slightly hydrate the seed to the point where germination processes commence, although they would indicate fast germination were re-imbibed under normal or stress situations (Singh et al., 2015). Seed priming enhances metabolic processes, preventing seed deterioration, breaking dormancy, and inducing systemic resistance to biotic and abiotic stresses (Pawar and Laware, 2018). Seed priming, owing to its efficiency and lack of the need for expensive equipment and chemicals, could be used as a simple method for overcoming problems associated with poor germination and seedling establishment, thereby assisting in the sustainability of agriculture and cost effective, economical, non-toxic and eco-friendly sources (Mishra et al., 2017).

Nutrients have a key function in enhancing pulse seed production (Chandrasekhar and Bangarusamy, 2003). Foliar application is associated with the advantages of rapid and effective nutrient use, reduction of losses due to leaching and fixation and aids in controlling nutrient uptake by plants (Manonmani and Srimathi, 2009). Micronutrient deficiencies in humans and crop plants are hard to detect. Therefore, the problem is referred to as "hidden hunger" (Stein et al., 2008). The zinc (Zn) deficiency is the most prevalent nutritional deficiency after iron and iodine. According to the world health organization, $\mathrm{Zn}$ deficiency is the fifth major cause of illness among juveniles and old age people in developing countries. The majority of the Indian soils are reported to be $\mathrm{Zn}$ deficient. Consequently, food crops cultivated in those soils contain a minimum level of $\mathrm{Zn}$ nutrient. Crop species have shown significant genetic variability in sustaining growth and yield in $\mathrm{Zn}$ deficient conditions.

"The art and science of altering materials at the nanoscale level is known as nanotechnology." Nanoparticles are metal particles that are spherical or faceted and are generally $100 \mathrm{~nm}$ in size. These nanoparticles have a high surface area $(30-50 \mathrm{~m} 2 / \mathrm{g})$, high activity, a better catalytic surface, a quick chemical reaction, are rapidly dispersible, and absorb a large amount of water. As a result, nano fertilizers may improve nutrient absorption efficiency, boost yield and nutrient content in edible portions, and reduce nutrient buildup in the soil (Saraswathi, 2019). Nanotechnology has been regarded as the "next great frontier of agricultural research" and it plays an important role in revolutionizing agriculture and food production through effective soil nutrient management (Subramanian and Tarafdar, 2011).

In this context, the aim of the present study was to develop cropping system along with bio seed priming of plant leaf extracts and foliar nanoparticles of zinc oxide nanoparticle which can be an effective and eco-friendly technique that could improve the faster emergence, more uniform plant population and increasing yield with improved seed nutrient content.

\section{MATERIALS AND METHODS}

The laboratory analysis was conducted at the Agronomy Department, Tamil Nadu Agricultural University (TNAU), Coimbatore, with the foremost aim to prepare botanical leaf extracts for seed priming using Neem (Azadirachta indica), Prosopis (Prosopis juliflora) and Pungam (Pogamia pinnata) leaves and synthesis of zinc oxide nanoparticles for foliar spray using chemical method. Thus, synthesized zinc oxide nanoparticles were characterized by using zeta potential. The average-sized zinc oxide nanoparticles were tested using Particle Size Analyzer (PSA), UV-Visible Spectroscopy (UV-Vis), Fourier Transform Infrared Spectroscopy (FT-IR), X-Ray Diffraction (XRD), Scanning Electron Microscope (SEM) and Transmission Electron Microscope (TEM) for shape and size and Stability of suspension and Energy Dispersive X-Ray Spectrosco- 
py (EDAX) available at Department of Nano Science \& Technology, Tamil Nadu Agricultural University, Coimbatore.

\section{Standardization of soaking duration and concentration of leaf extracts as priming agent for seed priming}

Fresh leaves of Neem (A. indica), Prosopis ( $P$. juliflora), and Pungam $(P$. pinnata) were picked and dried individually in the shade. Using a mortar and pestle, the dried leaves were crushed. Then, using a weighing scale, precisely weigh one gramme of leaf powder and dissolve it in $100 \mathrm{ml}$ of distilled water that was originally measured in the beaker to generate a 1 percent extract. To eliminate undesired material and leaf debris, the leaf extract was filtered through muslin cloth (Gunasekar et al., 2017).

The seeds of finger millet and greengram were primed by adopting the following Seed to solution ratio and seed soaking duration of finger millet (1:1 and 6 hours) and greengram (1:0.3 and 3 hours) already standardized as per Crop Production Guide (CPG, 2012), Department of Agriculture, Government of Tamil Nadu. The seeds were indeed air-dried in the shade to return to their normal moisture content before being tested for seed quality characteristics.

\section{Synthesis of $\mathrm{ZnO}$ nanoparticles}

$\mathrm{ZnO}$ nanoparticles (NPs) were synthesized by dissolving $0.02 \mathrm{M}$ aqueous Zinc acetate dihydrate in $50 \mathrm{ml}$ distilled water under vigorous stirring. At room temperature, aqueous $2.0 \mathrm{M} \mathrm{NaOH}$ was added drop by drop to reach $\mathrm{pH} 12$ and which was then placed in a magnetic stirrer for $2 \mathrm{hr}$. After completion of the reaction, the white precipitate formed was washed thoroughly with distilled water followed by ethanol to remove the impurities. The precipitate was dried overnight in a hot air oven at $60^{\circ} \mathrm{C}$. This drying procedure completely converted $\mathrm{Zn}(\mathrm{OH}) 2$ into $\mathrm{ZnO}$ nanoparticles.

The farm is geographically situated in the NorthWestern part of Tamil Nadu at $11^{\circ} \mathrm{N}$ latitude and $77^{\circ} \mathrm{E}$ longitude with an altitude of $426.72 \mathrm{~m}$ above mean sea level (MSL). Coimbatore is located in the Western Argo Climatic Zones of Tamil Nadu. The field experiments were carried out at field number '36 F' of Eastern Block Farm, Central farm unit, Tamil Nadu Agricultural University, Coimbatore during Kharif (June to August) seasons of 2020 to study the response of effective farming practice for sole finger millet and with greengram intercropping system under rainfed conditions to varying levels of bio-seed priming and nano zinc.

Finger millet variety $\mathrm{CO} 15$ was taken as the main crop in this study. This variety was released by the Centre of Excellence on Millets, Athiyandal, TNAU in 2013. It is a popular high yielding and long duration variety rich in protein (11.8 percent) and non-lodging strain with a duration of 125 days. Greengram variety $\mathrm{CO} 8$ was taken as the intercrop in this study. This variety was released by the Department of Pulses, TNAU, Coimbatore during 2013.

The following treatments schedules were used for conducting the field trail in kharif season of finger millet intercropping system to optimizing the suitable treatment combinations of bio seed priming and nano zinc oxide nanoparticles application for finger millet intercropping system. The field experiment was laid out in a factorial randomized block design (FRBD) with three replications. All the treatments and replications were randomized to reduce the experimental error. FACTOR - I (cropping system): $M_{1}$ - Sole finger millet and $M_{2}-$ Finger millet + Greengram (2:1) in main plot and FACTOR - II (Bio seed priming and Foliar ZnO nanoparticle): $S_{1}-$ Neem leaf extract 1 per cent alone, $S_{2}-$ Neem leaf extract 1 per cent alone + Foliar $\mathrm{ZnO}$ nanoparticle @ 250 ppm, $S_{3}-$ Neem leaf extract 1 per cent alone + Foliar ZnO nanoparticle @ 500 ppm, S ${ }_{4}$ Neem leaf extract 1 per cent alone + Foliar $\mathrm{ZnO}$ nanoparticle @ 750 ppm, $S_{5}-$ Neem leaf extract 1 per cent alone + Foliar ZnO nanoparticle @ 1000 ppm, $S_{6}$ Prosopis leaf extract 1 per cent alone, $S_{7}-$ Prosopis leaf extract 1 per cent alone + Foliar $\mathrm{ZnO}$ nanoparticle @ 250 ppm, $S_{8}-$ Prosopis leaf extract 1 per cent alone + Foliar ZnO nanoparticle @ 500 ppm, $S_{9}$ - Prosopis leaf extract 1 per cent alone + Foliar $\mathrm{ZnO}$ nanoparticle @ 750 ppm, $S_{10}$ - Prosopis leaf extract 1 per cent alone + Foliar ZnO nanoparticle @ 1000 ppm, $S_{11}$ Pungam leaf extract 1 per cent alone, $S_{12}$ - Pungam leaf extract 1 per cent alone + Foliar $\mathrm{ZnO}$ nanoparticle @ 250 ppm, $S_{13}-$ Pungam leaf extract 1 per cent alone + Foliar ZnO nanoparticle @ 500 ppm, $\mathrm{S}_{14}$ Pungam leaf extract 1 per cent alone + Foliar $\mathrm{ZnO}$ nanoparticle @ 750 ppm and $S_{15}$ - Pungam leaf extract 1 per cent alone + Foliar ZnO nanoparticle @ 1000 ppm. The treatments were randomly allotted to the plots as per the experimental design. *Foliar spray was done twice on 30 and 60 DAS.

\section{RESULTS AND DISCUSSION}

\section{Growth components}

The experiments with treatments showed $(\leq 0.05)$ a significant level of difference in main as well as in subplot treatments of observations presented in Tables 1 to 3. All the growth components viz., plant height at harvest, LAl at harvest, number of tiller $\mathrm{m}^{-2}$, DMP $(\mathrm{Kg}$ $\mathrm{ha}^{-1}$ ) at harvest, days to 50 per cent panicle initiation, days to 50 per cent flowering, days to physiological maturity, chlorophyll index (SPAD) at harvest, number of productive tillers $\mathrm{m}^{-2}$ and tiller conversion efficiency per cent showed superior performance with finger millet intercropped with greengram as compared to sole crop of finger millet. 
Table 1. Effect of bio-seed priming and nano zinc foliar spray on plant height $(\mathrm{cm})$, leaf area index (LAI) and number of tillers per $\mathrm{m}^{-2}$ of finger millet at harvest

\begin{tabular}{|c|c|c|c|c|c|c|c|c|c|}
\hline \multirow{2}{*}{ Treatments } & \multicolumn{3}{|c|}{ Plant height at harvest } & \multicolumn{3}{|c|}{ LAI at harvest } & \multicolumn{3}{|c|}{ No. of tillers per $\mathrm{m}^{-2}$ at harvest } \\
\hline & $M_{1}$ & $M_{2}$ & Mean & $M_{1}$ & $\mathbf{M}_{2}$ & Mean & $M_{1}$ & $\mathbf{M}_{2}$ & Mean \\
\hline$S_{1}$ & 129.45 & 133.72 & 131.59 & 3.35 & 3.46 & 3.40 & 114 & 120 & 117 \\
\hline $\mathrm{S}_{2}$ & 131.53 & 136.74 & 134.14 & 3.56 & 3.85 & 3.70 & 138 & 147 & 143 \\
\hline $\mathrm{S}_{3}$ & 145.62 & 148.94 & 147.28 & 5.40 & 5.67 & 5.53 & 199 & 212 & 205 \\
\hline $\mathrm{S}_{4}$ & 142.84 & 144.97 & 143.91 & 4.80 & 5.04 & 4.92 & 175 & 189 & 182 \\
\hline $\mathrm{S}_{5}$ & 136.64 & 141.07 & 138.86 & 4.06 & 4.53 & 4.30 & 154 & 164 & 159 \\
\hline $\mathrm{S}_{6}$ & 130.02 & 135.24 & 132.63 & 3.49 & 3.74 & 3.62 & 126 & 134 & 130 \\
\hline $\mathrm{S}_{7}$ & 135.76 & 139.49 & 137.63 & 3.90 & 4.21 & 4.06 & 150 & 159 & 155 \\
\hline $\mathrm{S}_{8}$ & 146.34 & 149.75 & 148.05 & 5.77 & 5.92 & 5.84 & 218 & 230 & 224 \\
\hline $\mathrm{S}_{9}$ & 144.21 & 147.28 & 145.75 & 5.21 & 5.52 & 5.36 & 194 & 205 & 200 \\
\hline$S_{10}$ & 138.04 & 143.64 & 140.84 & 4.59 & 4.85 & 4.72 & 163 & 174 & 168 \\
\hline$S_{11}$ & 129.93 & 134.95 & 132.44 & 3.42 & 3.62 & 3.52 & 122 & 131 & 127 \\
\hline $\mathrm{S}_{12}$ & 133.59 & 138.56 & 136.08 & 3.66 & 3.95 & 3.81 & 148 & 153 & 150 \\
\hline$S_{13}$ & 146.34 & 148.94 & 147.64 & 5.59 & 5.81 & 5.70 & 211 & 223 & 217 \\
\hline$S_{14}$ & 143.36 & 146.40 & 144.88 & 5.01 & 5.33 & 5.17 & 187 & 196 & 191 \\
\hline$S_{15}$ & 137.93 & 142.95 & 140.44 & 4.40 & 4.71 & 4.56 & 159 & 170 & 164 \\
\hline \multirow[t]{2}{*}{ Mean } & 138.11 & 142.18 & & 4.41 & 4.68 & & 164 & 174 & \\
\hline & SEd & $\begin{array}{l}C D \\
(0.05)\end{array}$ & & Sed & $C D$ & & SEd & $\mathrm{CD}(0.05)$ & \\
\hline$M$ & 1.879 & 3.761 & & 0.055 & 0.110 & & 2.146 & 4.296 & \\
\hline$S$ & 5.145 & 10.299 & & 0.151 & 0.302 & & 5.877 & 11.764 & \\
\hline$M \times S$ & 7.276 & NS & & 0.213 & NS & & 8.311 & NS & \\
\hline
\end{tabular}

Note: $M_{1}-$ Sole finger millet and $M_{2}-$ Finger millet + Greengram (2:1) in main plot and $S_{1}-$ Neem leaf extract 1 per cent alone, $S_{2}-$ Neem leaf extract 1 per cent alone + Foliar ZnO nanoparticle @ 250 ppm, $S_{3}$ - Neem leaf extract 1 per cent alone + Foliar ZnO nanoparticle @ 500 ppm, $S_{4}-$ Neem leaf extract 1 per cent alone + Foliar ZnO nanoparticle @ 750 ppm, $S_{5}-$ Neem leaf extract 1 per cent alone + Foliar ZnO nanoparticle @ 1000 ppm, $S_{6}$ - Prosopis leaf extract 1 per cent alone, $S_{7}$ - Prosopis leaf extract 1 per cent alone + Foliar ZnO nanoparticle @ 250 ppm, $\mathrm{S}_{8}$ - Prosopis leaf extract 1 per cent alone + Foliar ZnO nanoparticle @ 500 ppm, $\mathrm{S}_{9}-$ Prosopis leaf extract 1 per cent alone + Foliar ZnO nanoparticle @ 750 ppm, $\mathrm{S}_{10}$ - Prosopis leaf extract 1 per cent alone + Foliar ZnO nanoparticle @ 1000 ppm, $S_{11}$ - Pungam leaf extract 1 per cent alone, $S_{12}$ - Pungam leaf extract 1 per cent alone + Foliar ZnO nanoparticle @ 250 ppm, $S_{13}-$ Pungam leaf extract 1 per cent alone + Foliar ZnO nanoparticle @ 500 ppm, $S_{14}$ - Pungam leaf extract 1 per cent alone + Foliar ZnO nanoparticle @ 750 ppm and $\mathrm{S}_{15}$ - Pungam leaf extract 1 per cent alone + Foliar ZnO nanoparticle @ 1000 ppm.

Among the two-cropping system, finger millet + greengram (2:1) $\left(\mathrm{M}_{2}\right)$ recorded the highest mean of plant height at harvest of $(142.18 \mathrm{~cm})$, LAl at harvest (4.68), number of tiller $\mathrm{m}^{-2}(174)$, DMP $\left(9404 \mathrm{~kg} \mathrm{ha}^{-1}\right)$ at harvest, days to 50 per cent panicle initiation (71.99 DAS), days to 50 per cent flowering (79.21 DAS), days to physiological maturity (105.77 DAS), chlorophyll index (SPAD) at harvest (35.67), number of productive tiller $\mathrm{m}^{-2}$ (174.34), tiller conversion efficiency (100.68 per cent) followed by sole finger millet $\left(\mathrm{M}_{1}\right) 138.11 \mathrm{~cm}$, 4.41, 164, $9008 \mathrm{~kg} \mathrm{ha}^{-1}, 73.0$ DAS, 80.17 DAS,107.26 DAS, 34.30, 162.47, $99.18 \%$ at Plant height at harvest, $\mathrm{LAl}$ at harvest, number of tiller $\mathrm{m}^{-2}$, DMP $\left(\mathrm{kg} \mathrm{ha}^{-1}\right)$ at harvest, days to 50 per cent panicle initiation, days to 50 per cent flowering, days to physiological maturity, chlorophyll index (SPAD) at harvest, number of productive tiller $\mathrm{m}^{-2}$, tiller conversion efficiency percent given in Tables 2, 3 and 4, respectively.
Concerning bio seed priming and foliar zinc oxide nanoparticle spray, priming of Prosopis leaf extract of 1 per cent along with $500 \mathrm{ppm}$ of foliar $\mathrm{ZnO}$ nanoparticle $\left(\mathrm{S}_{8}\right)$ recorded higher plant height at harvest of $(148.05 \mathrm{~cm})$, LAI at harvest (5.84), number of tiller $\mathrm{m}^{-2}$ (224), DMP $\left(11350 \mathrm{~kg} \mathrm{ha}^{-1}\right)$ at harvest, days to 50 per cent panicle initiation (75.20 DAS), days to 50 per cent flowering (83.85 DAS), days to physiological maturity (110.50 DAS), chlorophyll index (SPAD) at harvest (39.27), number of productive tiller $\mathrm{m}^{-2}$ (222.40), tiller conversion efficiency (99.34 per cent). This was on par with Pungam leaf extract 1 per cent alone + foliar $\mathrm{ZnO}$ nanoparticle @ 500 ppm $\left(S_{13}\right)$ at all the stages of observation. The least growth components were obtained with Neem leaf extract 1 per cent alone $\left(S_{1}\right)$, the plant height at harvest, LAl at harvest, number of tiller $\mathrm{m}^{-2}$, DMP ( $\mathrm{kg}$ ha $\left.{ }^{-1}\right)$ at harvest, days to 50 per cent panicle initiation, days to 50 per cent flowering, days to physiological maturity, 
Mohanasundar, C. et al. / J. Appl. \& Nat. Sci. 13(4), 1552 - 1562 (2021)

Table 2. Effect of bio-seed priming and nano zinc foliar spray on DMP ( $\mathrm{Kg} \mathrm{ha}^{-1}$ ) at harvest, days to $50 \%$ panicle initiation, days to $50 \%$ flowering and physiological maturity of finger millet

\begin{tabular}{|c|c|c|c|c|c|c|c|c|c|c|c|c|}
\hline \multirow{2}{*}{ Treatments } & \multicolumn{3}{|c|}{$\begin{array}{c}\mathrm{DMP}\left(\mathrm{Kg} \mathrm{ha}^{-1}\right) \text { at } \\
\text { harvest }\end{array}$} & \multicolumn{3}{|c|}{$\begin{array}{l}\text { Days to } 50 \% \text { panicle } \\
\text { initiation }\end{array}$} & \multicolumn{3}{|c|}{$\begin{array}{l}\text { Days to } 50 \% \\
\text { flowering }\end{array}$} & \multicolumn{3}{|c|}{$\begin{array}{c}\text { Days to physiological } \\
\text { maturity }\end{array}$} \\
\hline & $\mathbf{M}_{1}$ & $\mathbf{M}_{2}$ & Mean & $\mathbf{M}_{1}$ & $\mathbf{M}_{2}$ & Mean & $\mathbf{M}_{1}$ & $\mathbf{M}_{2}$ & Mean & $\mathbf{M}_{1}$ & $\mathbf{M}_{2}$ & Mean \\
\hline$S_{1}$ & 7474 & 7712 & 7593 & 69.80 & 67.80 & 68.80 & 75.80 & 74.70 & 75.25 & 103.60 & 102.40 & 103.00 \\
\hline $\mathrm{S}_{2}$ & 7996 & 8296 & 8146 & 71.50 & 70.50 & 71.00 & 77.90 & 77.20 & 77.55 & 104.70 & 103.30 & 104.00 \\
\hline$S_{3}$ & 10272 & 10715 & 10493 & 74.80 & 74.30 & 74.55 & 83.10 & 81.70 & 82.40 & 110.20 & 108.50 & 109.35 \\
\hline$S_{4}$ & 9271 & 9827 & 9549 & 73.50 & 73.20 & 73.35 & 81.50 & 80.30 & 80.90 & 108.50 & 106.10 & 107.30 \\
\hline $\mathrm{S}_{5}$ & 8687 & 9105 & 8896 & 72.80 & 72.30 & 72.55 & 79.70 & 78.90 & 79.30 & 106.90 & 105.10 & 106.00 \\
\hline $\mathrm{S}_{6}$ & 7839 & 8022 & 7930 & 71.10 & 69.80 & 70.45 & 77.40 & 76.10 & 76.75 & 104.70 & 103.20 & 103.95 \\
\hline$S_{7}$ & 8471 & 8806 & 8638 & 72.70 & 71.20 & 71.95 & 78.90 & 78.50 & 78.70 & 106.10 & 104.30 & 105.20 \\
\hline $\mathrm{S}_{8}$ & 11138 & 11563 & 11350 & 75.50 & 74.90 & 75.20 & 84.80 & 82.90 & 83.85 & 111.10 & 109.90 & 110.50 \\
\hline $\mathrm{S}_{9}$ & 9898 & 10372 & 10135 & 74.60 & 73.60 & 74.10 & 82.30 & 81.60 & 81.95 & 109.80 & 107.80 & 108.80 \\
\hline$S_{10}$ & 9092 & 9567 & 9330 & 73.20 & 72.80 & 73.00 & 80.90 & 79.90 & 80.40 & 107.50 & 106.10 & 106.80 \\
\hline$S_{11}$ & 7781 & 7984 & 7883 & 70.60 & 68.00 & 69.30 & 76.10 & 75.80 & 75.95 & 103.80 & 102.90 & 103.35 \\
\hline$S_{12}$ & 8148 & 8585 & 8367 & 72.30 & 71.10 & 71.70 & 78.50 & 77.40 & 77.95 & 105.80 & 104.20 & 105.00 \\
\hline$S_{13}$ & 10593 & 11159 & 10876 & 75.20 & 74.50 & 74.85 & 83.50 & 82.30 & 82.90 & 110.20 & 109.50 & 109.85 \\
\hline$S_{14}$ & 9572 & 9996 & 9784 & 74.30 & 73.40 & 73.85 & 81.50 & 81.50 & 81.50 & 108.70 & 107.70 & 108.20 \\
\hline$S_{15}$ & 8887 & 9354 & 9121 & 73.10 & 72.50 & 72.80 & 80.60 & 79.30 & 79.95 & 107.30 & 105.60 & 106.45 \\
\hline \multirow[t]{2}{*}{ Mean } & 9008 & 9404 & & 73.00 & 71.99 & & 80.17 & 79.21 & & 107.26 & 105.77 & \\
\hline & SEd & CD (0.05) & & SEd & CD (0.05) & & SEd & $C D(0.05)$ & & SEd & CD (0.05) & \\
\hline M & 120.550 & 241.306 & & 1.111 & NS & & 1.125 & NS & & 1.402 & NS & \\
\hline$S$ & 330.139 & 660.844 & & 3.044 & NS & & 3.080 & NS & & 3.841 & NS & \\
\hline$M \times S$ & 466.887 & NS & & 4.304 & NS & & 4.356 & NS & & 5.431 & NS & \\
\hline
\end{tabular}

Note: $M_{1}-$ Sole finger millet and $M_{2}-$ Finger millet + Greengram (2:1) in main plot and $S_{1}-$ Neem leaf extract 1 per cent alone, $\mathrm{S}_{2}$ - Neem leaf extract 1 per cent alone + Foliar ZnO nanoparticle @ 250 ppm, $\mathrm{S}_{3}-$ Neem leaf extract 1 per cent alone + Foliar ZnO nanoparticle @ 500 ppm, $S_{4}-$ Neem leaf extract 1 per cent alone + Foliar ZnO nanoparticle @ 750 ppm, $S_{5}-$ Neem leaf extract 1 per cent alone + Foliar ZnO nanoparticle @ 1000 ppm, $S_{6}$ - Prosopis leaf extract 1 per cent alone, $S_{7}$ - Prosopis leaf extract 1 per cent alone + Foliar ZnO nanoparticle @ 250 ppm, S - Prosopis leaf extract 1 per cent alone + Foliar ZnO nanoparticle @ 500 ppm, $\mathrm{S}_{9}$ - Prosopis leaf extract 1 per cent alone + Foliar ZnO nanoparticle @ 750 ppm, $\mathrm{S}_{10}$ - Prosopis leaf extract 1 per cent alone + Foliar $\mathrm{ZnO}$ nanoparticle @ 1000 ppm, $\mathrm{S}_{11}-$ Pungam leaf extract 1 per cent alone, $\mathrm{S}_{12}$ - Pungam leaf extract 1 per cent alone + Foliar $\mathrm{ZnO}$ nanoparticle @ 250 ppm, $S_{13}$ - Pungam leaf extract 1 per cent alone + Foliar ZnO nanoparticle @ 500 ppm, $S_{14}$ - Pungam leaf extract 1 per cent alone + Foliar ZnO nanoparticle @ 750 ppm and $\mathrm{S}_{15}-$ Pungam leaf extract 1 per cent alone + Foliar ZnO nanoparticle @ 1000 ppm.

chlorophyll index (SPAD) at harvest, number of productive tiller $\mathrm{m}^{-2}$, tiller conversion efficiency per cent being $131.59 \mathrm{~cm}, 3.40,117,7593 \mathrm{~kg} \mathrm{ha}^{-1}, 68.80$ DAS, 75.25 DAS,103.0 DAS, 31.30, 115.36, 98.62 per cent, respectively. The interaction effect of cropping system and bio seed priming and foliar zinc nanoparticle spray was non-significant (at 0.05 level) irrespective of the growth stages stated in Tables 2-4, respectively.

The increased growth of finger millet with greengram may be due to the compensating impact of greengram, which provided nitrogen to finger millet and the greater usage of natural resources by the finger millet + greengram intercropping system. (Tripathi and Kushwaha, 2013) also reported that plant height and number of leaves per plant of pearl millet under intercropping system were either higher or statistically similar to sole pearl millet, which could be attributed to effective utili- sation of space and light interception as nutrient contribution of leguminous crop to cereal crop.

Finger millet intercropped with greengram had a beneficial influence on LAI at all stages of crop growth. This might be due to a larger tiller number, which led in a greater number of leaves, resulting in a greater LAI value. (Fawusi et al., 1982) also indicated that maizebased intercropping systems had a greater leaf area index than solo crops. (Kumar et al., 2008) observed that both little millet and pigeonpea sole crops produced greater total dry matter. They also found that a $6: 2$ row ratio led to an increased total dry matter production of little millet and pigeonpea. This might be attributed to the increased dry matter accumulation in the leaf, stem, and reproductive sections. Kaushik and Sharma (2017) reported similar findings in a wheatbased intercropping system noticed higher leaf area 
Table 3. Effect of bio-seed priming and nano zinc foliar spray on chlorophyll index (SPAD value) at harvest, number of productive tillers per hill, number of productive tillers per $\mathrm{m}^{-2}$ and tiller conversion efficiency (\%) of finger millet

\begin{tabular}{|c|c|c|c|c|c|c|c|c|c|}
\hline \multirow[b]{2}{*}{ Treatments } & \multicolumn{3}{|c|}{ Chlorophyll index at harvest } & \multicolumn{3}{|c|}{ No. of productive tillers per $\mathrm{m}^{-2}$} & \multicolumn{3}{|c|}{ Tiller conversion efficiency (\%) } \\
\hline & $\mathbf{M}_{1}$ & $\mathbf{M}_{2}$ & Mean & $\mathbf{M}_{1}$ & $\mathbf{M}_{2}$ & Mean & $M_{1}$ & $\mathbf{M}_{2}$ & Mean \\
\hline $\mathrm{S}_{1}$ & 30.26 & 32.34 & 31.30 & 112.32 & 118.40 & 115.36 & 98.32 & 98.93 & 98.62 \\
\hline $\mathrm{S}_{2}$ & 31.36 & 33.01 & 32.19 & 136.96 & 146.56 & 141.76 & 99.12 & 99.57 & 99.34 \\
\hline $\mathrm{S}_{3}$ & 36.60 & 39.55 & 38.07 & 198.40 & 210.88 & 204.64 & 99.68 & 99.55 & 99.61 \\
\hline $\mathrm{S}_{4}$ & 36.10 & 36.79 & 36.44 & 173.12 & 188.16 & 180.64 & 99.16 & 99.32 & 99.24 \\
\hline $\mathrm{S}_{5}$ & 33.76 & 33.82 & 33.79 & 152.96 & 162.24 & 157.60 & 99.42 & 99.22 & 99.32 \\
\hline $\mathrm{S}_{6}$ & 31.02 & 32.75 & 31.89 & 124.48 & 132.80 & 128.64 & 98.98 & 99.28 & 99.13 \\
\hline$S_{7}$ & 33.48 & 33.61 & 33.55 & 148.80 & 157.76 & 153.28 & 99.15 & 99.20 & 99.17 \\
\hline $\mathrm{S}_{8}$ & 38.15 & 40.38 & 39.27 & 215.36 & 229.44 & 222.40 & 98.83 & 99.86 & 99.34 \\
\hline $\mathrm{S}_{9}$ & 36.28 & 37.62 & 36.95 & 192.32 & 203.84 & 198.08 & 99.17 & 99.38 & 99.28 \\
\hline$S_{10}$ & 35.76 & 36.45 & 36.10 & 160.96 & 172.16 & 166.56 & 98.90 & 99.08 & 98.99 \\
\hline$S_{11}$ & 30.76 & 32.51 & 31.64 & 121.28 & 156.80 & 139.04 & 99.21 & 119.51 & 109.36 \\
\hline$S_{12}$ & 33.06 & 33.25 & 33.16 & 147.20 & 152.00 & 149.60 & 99.39 & 99.58 & 99.49 \\
\hline$S_{13}$ & 37.58 & 39.94 & 38.76 & 208.64 & 221.12 & 214.88 & 99.09 & 99.14 & 99.11 \\
\hline$S_{14}$ & 36.25 & 37.16 & 36.71 & 186.24 & 193.92 & 190.08 & 99.83 & 99.18 & 99.51 \\
\hline$S_{15}$ & 34.12 & 35.82 & 34.97 & 158.08 & 168.96 & 163.52 & 99.48 & 99.44 & 99.46 \\
\hline \multirow[t]{2}{*}{ Mean } & 34.30 & 35.67 & & 162.47 & 174.34 & & 99.18 & 100.68 & \\
\hline & SEd & $\begin{array}{c}C D \\
(0.05)\end{array}$ & & Sed & $\begin{array}{c}C D \\
(0.05)\end{array}$ & & SEd & $\begin{array}{c}C D \\
(0.05)\end{array}$ & \\
\hline M & 0.422 & 0.886 & & 2.152 & 4.308 & & 1.803 & NS & \\
\hline$S$ & 1.212 & 2.425 & & 5.894 & 11.798 & & 4.938 & NS & \\
\hline$M \times S$ & 1.714 & NS & & 8.336 & NS & & 6.984 & NS & \\
\hline
\end{tabular}

Note: $M_{1}-$ Sole finger millet and $M_{2}-$ Finger millet + Greengram (2:1) in main plot and $S_{1}-$ Neem leaf extract 1 per cent alone, $S_{2}-$ Neem leaf extract 1 per cent alone + Foliar ZnO nanoparticle @ 250 ppm, $S_{3}$ - Neem leaf extract 1 per cent alone + Foliar ZnO nanoparticle @ 500 ppm, S $S_{4}$ Neem leaf extract 1 per cent alone + Foliar ZnO nanoparticle @ 750 ppm, $S_{5}-$ Neem leaf extract 1 per cent alone + Foliar ZnO nanoparticle @ 1000 ppm, $S_{6}$ - Prosopis leaf extract 1 per cent alone, $\mathrm{S}_{7}$ - Prosopis leaf extract 1 per cent alone +

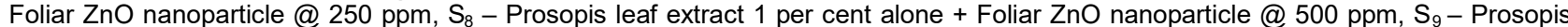
leaf extract 1 per cent alone + Foliar ZnO nanoparticle @ 750 ppm, $S_{10}$ - Prosopis leaf extract 1 per cent alone + Foliar ZnO nanoparticle @ 1000 ppm, $S_{11}-$ Pungam leaf extract 1 per cent alone, $S_{12}$ - Pungam leaf extract 1 per cent alone + Foliar ZnO nanoparticle @ 250 ppm, $S_{13}-$ Pungam leaf extract 1 per cent alone + Foliar ZnO nanoparticle @ 500 ppm, $S_{14}$ - Pungam leaf extract 1 per cent alone + Foliar ZnO nanoparticle @ 750 ppm and $\mathrm{S}_{15}$ - Pungam leaf extract 1 per cent alone + Foliar ZnO nanoparticle @ 1000 ppm.

index compare to sole wheat cropping system.

Prosopis leaf extract of 1 percent alone with 500 ppm of foliar $\mathrm{ZnO}$ nanoparticle (S8) in sorghum recorded higher growth parameters compared to higher concentration (1000 ppm) due to enhance in plant height and photosynthetically active leaf area due to nano $\mathrm{ZnO}$ might have been the reason for increased dry matter accumulation and could be due to the complementary effect of other innate nutrients like magnesium, iron, and sulphur with zinc (Koti et al., 2009; Poornima and Koti, 2019).

Zinc acts as an enzyme activator in plants and is directly involved in the biosynthesis of auxin, which generates more cells and dry matter that could be stored in seeds, as stated in various crop experiments by (Slaton et al., 2001; Rehman et al., 2002) in rice, (Genc et al.,
2006; Ozkutlu et al., 2006) in bread wheat and (Anand $R, 2008$ ) in rabi season sorghum crop. Prasad et al. (2012) demonstrated that nano $\mathrm{ZnO}$ increased seed germination, seedling vigour, early flowering, and leaf chlorophyll content. They also found beneficial effects of NPs in enhancing plant growth, development, and yield in peanuts at low concentrations, but at larger concentrations, $\mathrm{ZnO}$ NPs were negative, exactly like the bulk nutrients.

\section{Yield attributes}

The present investigation showed that significant difference in main as well as sub treatments of observations given in Tables 4 and 5 . All the yield attributes viz., earhead weight $(\mathrm{g})$, earhead length $(\mathrm{cm})$, number of fingers per earhead, finger length $(\mathrm{cm})$, number of 
Mohanasundar, C. et al. / J. Appl. \& Nat. Sci. 13(4), 1552 - 1562 (2021)

Table 4. Effect of bio-seed priming and nano zinc foliar spray on earhead weight ( $\mathrm{g}$ ), earhead length (cm), number of fingers per earhead and finger length $(\mathrm{cm})$ of finger millet

\begin{tabular}{|c|c|c|c|c|c|c|c|c|c|c|c|c|}
\hline \multirow{2}{*}{ Treatments } & \multicolumn{3}{|c|}{ Earhead weight (g) } & \multicolumn{3}{|c|}{ Earhead length $(\mathrm{cm})$} & \multicolumn{3}{|c|}{$\begin{array}{l}\text { No. of fingers per } \\
\text { earhead }\end{array}$} & \multicolumn{3}{|c|}{ Finger length $(\mathrm{cm})$} \\
\hline & $\overline{M_{1}}$ & $\mathbf{M}_{2}$ & Mean & $M_{1}$ & $\mathbf{M}_{2}$ & Mean & $M_{1}$ & $M_{2}$ & Mean & $M_{1}$ & $M_{2}$ & Mean \\
\hline $\mathrm{S}_{1}$ & 5.15 & 5.47 & 5.31 & 7.14 & 7.62 & 7.38 & 6.42 & 6.96 & 6.69 & 6.63 & 7.12 & 6.88 \\
\hline $\mathrm{S}_{2}$ & 5.49 & 5.94 & 5.72 & 7.81 & 7.97 & 7.89 & 7.03 & 7.61 & 7.32 & 7.36 & 7.62 & 7.49 \\
\hline $\mathrm{S}_{3}$ & 6.52 & 6.74 & 6.63 & 10.59 & 11.37 & 10.98 & 8.39 & 8.44 & 8.42 & 9.13 & 9.57 & 9.35 \\
\hline $\mathrm{S}_{4}$ & 6.29 & 6.46 & 6.38 & 9.32 & 10.06 & 9.69 & 8.12 & 8.26 & 8.19 & 8.29 & 8.36 & 8.32 \\
\hline $\mathrm{S}_{5}$ & 5.82 & 6.21 & 6.02 & 8.55 & 8.85 & 8.70 & 7.62 & 7.90 & 7.76 & 7.83 & 7.96 & 7.90 \\
\hline $\mathrm{S}_{6}$ & 5.36 & 5.85 & 5.61 & 7.71 & 7.94 & 7.82 & 6.87 & 7.32 & 7.10 & 7.33 & 7.58 & 7.46 \\
\hline $\mathrm{S}_{7}$ & 5.65 & 6.18 & 5.92 & 8.19 & 8.66 & 8.42 & 7.59 & 7.77 & 7.68 & 7.61 & 7.78 & 7.69 \\
\hline $\mathrm{S}_{8}$ & 6.73 & 6.89 & 6.81 & 11.45 & 11.72 & 11.59 & 8.46 & 8.73 & 8.60 & 9.68 & 9.94 & 9.81 \\
\hline $\mathrm{S}_{9}$ & 6.48 & 6.69 & 6.59 & 10.44 & 10.87 & 10.66 & 8.31 & 8.42 & 8.37 & 8.72 & 8.88 & 8.80 \\
\hline$S_{10}$ & 6.04 & 6.35 & 6.20 & 9.18 & 9.23 & 9.20 & 7.96 & 8.20 & 8.08 & 8.16 & 8.25 & 8.21 \\
\hline$S_{11}$ & 5.24 & 5.73 & 5.49 & 7.36 & 7.88 & 7.62 & 6.62 & 7.11 & 6.87 & 6.89 & 7.31 & 7.10 \\
\hline$S_{12}$ & 5.53 & 6.03 & 5.78 & 8.04 & 8.51 & 8.27 & 7.20 & 7.69 & 7.45 & 7.57 & 7.74 & 7.65 \\
\hline $\mathrm{S}_{13}$ & 6.64 & 6.81 & 6.73 & 11.15 & 11.44 & 11.30 & 8.40 & 8.52 & 8.46 & 9.43 & 9.72 & 9.58 \\
\hline $\mathrm{S}_{14}$ & 6.37 & 6.55 & 6.46 & 9.65 & 10.58 & 10.12 & 8.27 & 8.34 & 8.31 & 8.45 & 8.64 & 8.55 \\
\hline$S_{15}$ & 5.99 & 6.27 & 6.13 & 8.87 & 8.94 & 8.91 & 7.81 & 8.06 & 7.94 & 8.04 & 8.14 & 8.09 \\
\hline \multirow[t]{2}{*}{ Mean } & 5.95 & 6.28 & & 9.03 & 9.44 & & 7.67 & 7.96 & & 8.07 & 8.31 & \\
\hline & SEd & CD $(0.05$ & & SEd & $C D(0.05)$ & & SEd & CD (0.05) & & SEd & CD $(0.05$ & \\
\hline$M$ & 0.064 & 0.128 & & 0.129 & 0.258 & & 0.101 & 0.203 & & 0.113 & 0.226 & \\
\hline$S$ & 0.175 & 0.350 & & 0.353 & 0.706 & & 0.278 & 0.556 & & 0.309 & 0.619 & \\
\hline$M \times S$ & 0.248 & NS & & 0.499 & NS & & 0.393 & NS & & 0.437 & NS & \\
\hline
\end{tabular}

grains per earhead and thousand grain weight $(\mathrm{g})$ showed superior performance with finger millet intercropped with greengram as compared to sole crop of finger millet cultivation.

Among the two-cropping system, finger millet + greengram (2:1) $\left(\mathrm{M}_{2}\right)$ gave the highest mean of $6.28 \mathrm{~g}, 9.44$ $\mathrm{cm}, 7.96,8.31 \mathrm{~cm}, 2552.79$ and $2.51 \mathrm{~g}$ at earhead weight, earhead length, number of fingers per earhead, finger length, number of grains per earhead and thousand grain weight followed by sole finger millet of earhead weight $(5.95 \mathrm{~g})$, earhead length $(9.03 \mathrm{~cm})$, number of fingers per earhead (7.67) and finger length $(8.07 \mathrm{~cm})$, number of grains per earhead (2366.96) and thousand grain weight $(2.46 \mathrm{~g})$ specified in Tables 5 and 6 respectively.

With respect to bio seed priming and foliar zinc oxide nanoparticle spray, priming of Prosopis leaf extract 1 per cent alone along with 500 ppm of foliar $\mathrm{ZnO}$ nanoparticle $\left(S_{8}\right)$ recorded higher earhead weight $(6.81 \mathrm{~g})$, earhead length $(11.59 \mathrm{~cm})$, number of fingers per ear- head (8.60) and finger length $(9.81 \mathrm{~cm})$, number of grains per earhead (2464.11) and thousand grain weight $(2.85 \mathrm{~g})$. This was on par with Pungam leaf extract 1 per cent alone + foliar ZnO nanoparticle @ 500 ppm $\left(S_{13}\right)$ at all the stages of observation. The least yield components and yield were obtained with Neem leaf extract $1 \%$ alone $\left(S_{1}\right)$, the earhead weight $(g)$, earhead length $(\mathrm{cm})$, number of fingers per earhead, finger length $(\mathrm{cm})$, number of grains per earhead and thousand grain weight $(\mathrm{g})$ being $5.31 \mathrm{~g}, 7.38 \mathrm{~cm}, 6.69,6.88$ $\mathrm{cm}, 2277.45$ and $2.14 \mathrm{~g}$ respectively. The interaction effect of cropping system with bio seed priming and foliar zinc nanoparticle spray was non-significant at all the growth stages given in Tables 5 and 6, respectively. The highest dry matter weight was recorded when Prosopis leaf extract was primed with 1 per cent with 500 ppm of foliar ZnO nanoparticles (S8). A foliar application of nano $\mathrm{ZnO}$ resulted in increased dry matter accumulation due to increased plant height, leaves, and leaf area per plant. 
Table 5. Effect of bio-seed priming and nano zinc foliar spray on number of grains per earhead and thousand grain weight $(\mathrm{g})$ of finger millet

\begin{tabular}{|c|c|c|c|c|c|c|}
\hline \multirow[b]{2}{*}{ Treatments } & \multicolumn{3}{|c|}{ Number of grains per earhead } & \multicolumn{3}{|c|}{ Thousand grain weight $(\mathrm{g})$} \\
\hline & $\overline{M_{1}}$ & $\mathbf{M}_{2}$ & Mean & $\mathbf{M}_{1}$ & $\mathbf{M}_{2}$ & Mean \\
\hline $\mathrm{S}_{1}-$ Neem leaf extract $1 \%$ alone & 2148.52 & 2406.39 & 2277.45 & 2.12 & 2.15 & 2.14 \\
\hline $\begin{array}{l}\mathrm{S}_{2}-\text { Neem leaf extract } 1 \% \text { + Foliar Nano ZnO @ } \\
250 \text { ppm }\end{array}$ & 2278.28 & 2523.63 & 2400.95 & 2.26 & 2.28 & 2.27 \\
\hline $\begin{array}{l}\mathrm{S}_{3}-\text { Neem leaf extract 1\% + Foliar Nano ZnO @ } \\
500 \text { ppm }\end{array}$ & 2477.52 & 2652.91 & 2565.22 & 2.71 & 2.80 & 2.76 \\
\hline $\begin{array}{l}\mathrm{S}_{4}-\text { Neem leaf extract } 1 \% \text { + Foliar Nano ZnO @ } \\
750 \text { ppm }\end{array}$ & 2419.19 & 2593.75 & 2506.47 & 2.58 & 2.64 & 2.61 \\
\hline $\begin{array}{l}\mathrm{S}_{5}-\text { Neem leaf extract 1\% + Foliar Nano ZnO @ } \\
1000 \text { ppm }\end{array}$ & 2384.23 & 2553.30 & 2468.77 & 2.38 & 2.41 & 2.39 \\
\hline $\mathrm{S}_{6}-$ Prosopis leaf extract $1 \%$ alone & 2227.59 & 2476.01 & 2351.80 & 2.20 & 2.24 & 2.22 \\
\hline $\begin{array}{l}\mathrm{S}_{7}-\text { Prosopis leaf extract 1\% + Foliar Nano ZnO @ } \\
250 \text { ppm }\end{array}$ & 2320.45 & 2551.13 & 2435.79 & 2.32 & 2.35 & 2.34 \\
\hline $\begin{array}{l}\mathrm{S}_{8}-\text { Prosopis leaf extract 1\% + Foliar Nano ZnO @ } \\
500 \text { ppm }\end{array}$ & 2537.53 & 2390.70 & 2464.11 & 2.80 & 2.89 & 2.85 \\
\hline $\begin{array}{l}\mathrm{S}_{9}-\text { Prosopis leaf extract 1\% + Foliar Nano ZnO @ } \\
750 \text { ppm }\end{array}$ & 2461.24 & 2648.52 & 2554.88 & 2.69 & 2.75 & 2.72 \\
\hline $\begin{array}{l}S_{10}-\text { Prosopis leaf extract } 1 \% \text { + Foliar Nano ZnO @ } \\
1000 \text { ppm }\end{array}$ & 2408.09 & 2588.86 & 2498.48 & 2.51 & 2.59 & 2.55 \\
\hline $\mathrm{S}_{11}-$ Pongamia leaf $1 \%$ alone & 2204.53 & 2474.83 & 2339.68 & 2.17 & 2.19 & 2.18 \\
\hline $\begin{array}{l}\mathrm{S}_{12}-\text { Pongamia leaf extract 1\% + Foliar Nano ZnO } \\
\text { @ } 250 \text { ppm }\end{array}$ & 2286.89 & 2542.86 & 2414.87 & 2.27 & 2.30 & 2.29 \\
\hline $\begin{array}{l}\mathrm{S}_{13}-\text { Pongamia leaf extract } 1 \% \text { + Foliar Nano } \mathrm{ZnO} \\
@ 500 \text { ppm }\end{array}$ & 2494.00 & 2727.37 & 2610.69 & 2.76 & 2.84 & 2.80 \\
\hline $\begin{array}{l}S_{14}-\text { Pongamia leaf extract 1\% + Foliar Nano ZnO } \\
\text { @ } 750 \text { ppm }\end{array}$ & 2454.84 & 2607.97 & 2531.40 & 2.65 & 2.72 & 2.69 \\
\hline $\begin{array}{l}\text { S }_{15} \text { - Pongamia leaf extract 1\% + Foliar Nano ZnO } \\
@ 1000 \text { ppm }\end{array}$ & 2401.49 & 2553.63 & 2477.56 & 2.44 & 2.53 & 2.48 \\
\hline Mean & $\begin{array}{l}2366.96 \\
\text { SEd }\end{array}$ & $\begin{array}{l}2552.79 \\
C D(0.05)\end{array}$ & & $\begin{array}{l}2.46 \\
\text { SEd }\end{array}$ & $\begin{array}{l}2.51 \\
C D(0.05)\end{array}$ & \\
\hline Cropping systems (M) & 34.380 & 68.820 & & 0.044 & NS & \\
\hline Bio seed priming \& Foliar Nano ZnO Spray (S) & 94.155 & 188.471 & & 0.121 & 0.243 & \\
\hline $\mathrm{M} \times \mathrm{S}$ & 133.155 & NS & & 0.172 & NS & \\
\hline
\end{tabular}

Note: $M_{1}-$ Sole finger millet and $M_{2}-$ Finger millet + Greengram (2:1) in main plot and $S_{1}-$ Neem leaf extract 1 per cent alone, $S_{2}-$ Neem leaf extract 1 per cent alone + Foliar ZnO nanoparticle @ 250 ppm, $S_{3}$ - Neem leaf extract 1 per cent alone + Foliar ZnO nanoparticle @ 500 ppm, $S_{4}-$ Neem leaf extract 1 per cent alone + Foliar ZnO nanoparticle @ 750 ppm, S $5-$ Neem leaf extract 1 per cent alone + Foliar ZnO nanoparticle

\section{Yield}

Grain yield, finger millet equivalent yield and straw yield

The present investigation showed a significant difference in main as well as sub treatments of observations given in Table 6 and Fig. 1. Accordingly, the grain yield, finger millet equivalent yield and straw yield showed superior performance with finger millet intercropped greengram as compared to sole crop of finger millet alone. Among the two-cropping system, sole finger millet $\left(M_{1}\right)$ gave the highest mean finger millet grain and straw yield of $3040.23 \mathrm{~kg} \mathrm{ha}^{-1}$ and $6854.12 \mathrm{~kg} \mathrm{ha}^{-1}$ as compared to finger millet intercropping with greengram. Finger millet intercropping yield $\left(\mathrm{M}_{2}\right)$ is converted into finger millet equivalent yield (3200.88 $\left.\mathrm{kg} \mathrm{ha}^{-1}\right)$ obtain highest grain yield compared to sole finger millet (3040.23 kg ha ${ }^{-1}$ ), respectively.

With respect to bio seed priming and foliar zinc oxide nanoparticle spray, Prosopis leaf extract 1 per cent alone + Foliar ZnO nanoparticle @ 500 ppm $\left(\mathrm{S}_{8}\right)$ recorded higher grain yield (3238.84 $\left.\mathrm{kg} \mathrm{ha}^{-1}\right)$, finger millet equivalent yield $\left(3483.28 \mathrm{~kg} \mathrm{ha}^{-1}\right)$, straw yield $(7393.83$ $\left.\mathrm{kg} \mathrm{ha}^{-1}\right)$. This was on par with Pungam leaf extract $1 \%$ alone + foliar ZnO nanoparticle @ 500 ppm $\left(\mathrm{S}_{13}\right)$. The least yield components were obtained with Neem leaf

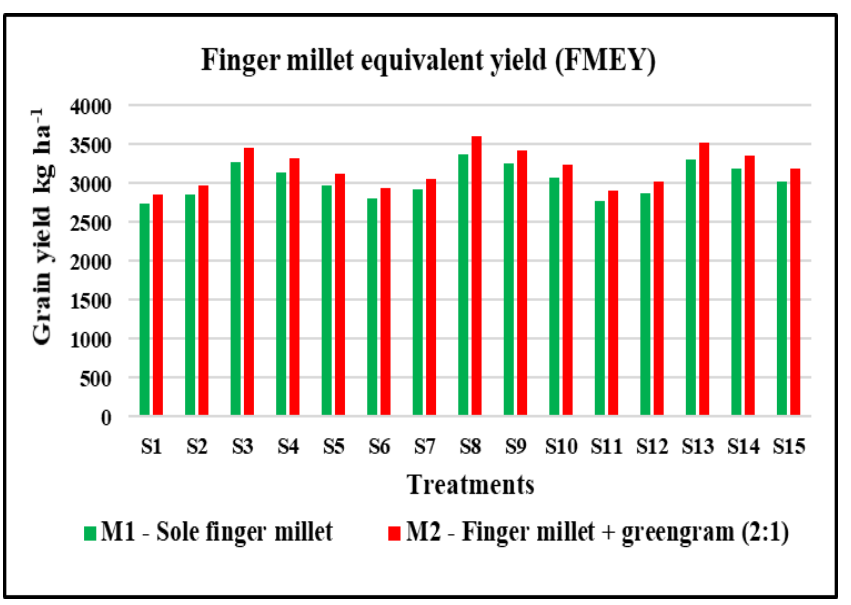

Fig. 1. Effect of bio seed priming and nano zinc foliar spray on FMEY of solo finger millet and intercropping of Finger millet + greengram 
Mohanasundar, C. et al. / J. Appl. \& Nat. Sci. 13(4), 1552 - 1562 (2021)

Table 6. Effect of bio-seed priming and nano zinc foliar spray on grain yield, finger millet equivalent yield (FMEY) and straw yield $\left(\mathrm{kg} \mathrm{ha}^{-1}\right)$ of finger millet

\begin{tabular}{|c|c|c|c|c|c|c|c|c|c|}
\hline \multirow{2}{*}{ Treatments } & \multicolumn{3}{|c|}{ Grain yield $\left(\mathrm{kg} \mathrm{ha}^{-1}\right)$} & \multicolumn{3}{|c|}{ Finger millet equivalent yield } & \multicolumn{3}{|c|}{ Straw yield $\left(\mathrm{kg} \mathrm{ha}^{-1}\right)$} \\
\hline & $\overline{M_{1}}$ & $\mathbf{M}_{2}$ & Mean & $\mathbf{M}_{1}$ & $\mathbf{M}_{2}$ & Mean & $M_{1}$ & $M_{2}$ & Mean \\
\hline $\mathrm{S}_{1}$ & 2746.73 & 2504.28 & 2625.51 & 2746.73 & 2862.99 & 2804.86 & 5795.61 & 4657.96 & 5226.78 \\
\hline $\mathrm{S}_{2}$ & 2850.61 & 2580.54 & 2715.57 & 2850.61 & 2970.11 & 2910.36 & 6157.31 & 4903.02 & 5530.17 \\
\hline $\mathrm{S}_{3}$ & 3280.86 & 2995.17 & 3138.01 & 3280.86 & 3462.56 & 3371.71 & 7710.02 & 6312.98 & 7011.50 \\
\hline $\mathrm{S}_{4}$ & 3141.10 & 2876.65 & 3008.87 & 3141.10 & 3327.05 & 3234.07 & 7193.11 & 5855.17 & 6524.14 \\
\hline $\mathrm{S}_{5}$ & 2970.62 & 2692.81 & 2831.72 & 2970.62 & 3119.96 & 3045.29 & 6654.19 & 5304.84 & 5979.52 \\
\hline $\mathrm{S}_{6}$ & 2812.39 & 2562.31 & 2687.35 & 2812.39 & 2946.51 & 2879.45 & 6001.64 & 4791.51 & 5396.58 \\
\hline$S_{7}$ & 2928.20 & 2647.69 & 2787.94 & 2928.20 & 3061.86 & 2995.03 & 6471.33 & 5162.99 & 5817.16 \\
\hline $\mathrm{S}_{8}$ & 3367.15 & 3110.53 & 3238.84 & 3367.15 & 3599.40 & 3483.28 & 8100.02 & 6687.65 & 7393.83 \\
\hline $\mathrm{S}_{9}$ & 3258.57 & 2961.15 & 3109.86 & 3258.57 & 3424.53 & 3341.55 & 7559.89 & 6176.68 & 6868.28 \\
\hline$S_{10}$ & 3066.14 & 2802.41 & 2934.27 & 3066.14 & 3242.52 & 3154.33 & 6960.14 & 5688.89 & 6324.52 \\
\hline$S_{11}$ & 2781.48 & 2532.47 & 2656.97 & 2781.48 & 2907.28 & 2844.38 & 5924.55 & 4786.36 & 5355.45 \\
\hline $\mathrm{S}_{12}$ & 2874.82 & 2614.31 & 2744.56 & 2874.82 & 3024.45 & 2949.64 & 6267.11 & 5019.47 & 5643.29 \\
\hline $\mathrm{S}_{13}$ & 3309.06 & 3050.96 & 3180.01 & 3309.06 & 3528.64 & 3418.85 & 7809.39 & 6501.02 & 7155.21 \\
\hline $\mathrm{S}_{14}$ & 3196.46 & 2900.28 & 3048.37 & 3196.46 & 3352.91 & 3274.69 & 7383.83 & 6006.47 & 6695.15 \\
\hline$S_{15}$ & 3019.30 & 2749.01 & 2884.15 & 3019.30 & 3182.41 & 3100.85 & 6823.61 & 5498.01 & 6160.81 \\
\hline \multirow[t]{2}{*}{ Mean } & 3040.23 & 2772.04 & & 3040.23 & 3200.88 & & 6854.12 & 5556.87 & \\
\hline & SEd & CD (0.05) & & Sed & CD (0.05) & & SEd & $C D(0.05)$ & \\
\hline M & 33.963 & 67.984 & & 39.503 & 79.074 & & 96.629 & 193.423 & \\
\hline S & 93.011 & 186.183 & & 108.184 & 216.553 & & 264.629 & 529.712 & \\
\hline$M \times S$ & 131.538 & NS & & 152.995 & NS & & 374.241 & NS & \\
\hline
\end{tabular}

Note: $\mathrm{M}_{1}-$ Sole finger millet and $\mathrm{M}_{2}-$ Finger millet + Greengram (2:1) in main plot and $\mathrm{S}_{1}-$ Neem leaf extract 1 per cent alone, $\mathrm{S}_{2}-$ Neem leaf extract 1 per cent alone + Foliar ZnO nanoparticle @ 250 ppm, $S_{3}-$ Neem leaf extract 1 per cent alone + Foliar ZnO nanoparticle@ 500 ppm, $S_{4}-$ Neem leaf extract 1 per cent alone + Foliar ZnO nanoparticle @ 750 ppm, S - Neem leaf extract 1 per cent alone + Foliar ZnO nanoparticle @ 1000 ppm, $\mathrm{S}_{6}$ - Prosopis leaf extract 1 per cent alone, $\mathrm{S}_{7}$ - Prosopis leaf extract 1 per cent alone + Foliar ZnO nanoparticle @ 250 ppm, $\mathrm{S}_{8}-$ Prosopis leaf extract 1 per cent alone + Foliar ZnO nanoparticle @ 500 ppm, S - Prosopis leaf extract 1 per cent alone + Foliar ZnO nanoparticle @ 750 ppm, S 10 - Prosopis leaf extract 1 per cent alone + Foliar ZnO nanoparticle @ 1000 ppm, $S_{11}$ - Pungam leaf extract 1 per cent alone, $S_{12}$ - Pungam leaf extract 1 per cent alone + Foliar ZnO nanoparticle @ 250 ppm, $S_{13}-$ Pungam leaf extract 1 per cent alone + Foliar ZnO nanoparticle @ 500 ppm, S 14 - Pungam leaf extract 1 per cent alone + Foliar ZnO nanoparticle @ 750 ppm and $\mathrm{S}_{15}$ - Pungam leaf extract 1 per cent alone + Foliar ZnO nanoparticle @ 1000 ppm.

extract $1 \%$ alone $\left(S_{1}\right)$. The interaction effect of cropping system and bio seed priming and foliar zinc nanoparticle spray was non-significant irrespective of all the growth stages.

Finger millet intercropped with greengram yielded comparable yields to finger millet grown as a sole crop. (Tripathi and Kushwaha, 2013) reported that yield attributes of pearl millet with intercrop viz., seed per panicle and 100 seed weight were significantly higher than that of sole pearl (Dass and Sudhishri, 2010) recorded higher system yield of finger millet in intercropped stand with pulses due to efficient use of nutrients, moisture, light and space in intercropped situation. An experimental study suggests that nano zinc oxide in a foliar spray can result in higher grain yields than normal fertilizer. Nanoparticles with a large surface area and small size (Mazaherinia et al., 2010) are expected to be the best material for use as zinc fertilizer in plants. As a result, when materials are transformed to a nanoscale, they change their physical, chemical, and biological 
properties, as well as catalytic properties and even become more active chemically and biologically (Prasad et al., 2012) investigated the impacts of nanoscale zinc oxide on peanut germination, growth, and yield and found dramatically increased growth and yield. (Reynolds, 2002) proved that micronutrients in the form of nanoparticles may be utilized to boost output in agricultural production.

Because of its nano size, high surface-to-volume ratio, and high reactivity, $\mathrm{ZnO}$ is rapidly absorbed by the leaf surface and metabolised faster than bulk form. Similar to our findings, nano $\mathrm{ZnO}$ showed significantly higher crop improvement by improving initial crop establishment, chlorophyll content, and ultimately crop growth and yield in the studies of Pandey et al. (2010) in Cicer arietinum, Boonyanitipong et al. (2011) in rice, Prasad et al., (2012) in peanuts, Sedghi et al. (2013) in soybean, Jayarambabu et al. (2014) in mungbean, Yang et al. (2015) in maize and rice and Poornima and Koti (2019) in sorghum. Prasad et al. (2012) stated that foliar application of zinc oxide nano particle is more effective than soil application and that zinc oxide foliar treatment improved pod yield and zinc content in peanut.

\section{Conclusion}

The study found that finger millet intercropped with greengram (2:1) performed better when combined with priming of Prosopis leaf extract of $1 \%$ alone together along with 500 ppm of foliar $\mathrm{ZnO}$ nanoparticle recorded greater growth parameters, resulting in an increase in finger millet crop yield. As a result, utilizing a small amount of fertiliser may minimise fertiliser application dosages, fertiliser waste, environmental dangers, and boost nutrient usage efficiency. As a result, the above treatment might be advised to rainfed millets farmers in order to boost productivity and obtain significant economic benefits from enhanced soil fertility. There is a need to explore the standardizing of nano fertiliser dosages for different crops and the ideal stage of crop development in order to produce improved crop output. There is also a need to understand the cellular mechanisms involved in nanoparticle absorption and translocation.

\section{Conflict of interest}

The authors declare that they have no conflict of interest.

\section{REFERENCES}

1. Ajibola, A. \& G Kolawole. (2019). Agronomic Evaluation of Performance of Sesame Varieties in Maize-based Intercropping System in the Southern Guinean Savanna of Nige- ria. Journal of Experimental Agriculture International,1-10. DOI:10.9734/jeai/2019/v37i330269

2. Anand R., K. R., Kamatar, M. Y., Mummigatti, U. V. \& Basavaraj, B. (2008). Evaluation of rabi sorghum genotypes for seed zinc content and yield in high regimes. Karnataka Journal of Agricultural Sciences 21 (04), 568-569.

3. Boonyanitipong, P., P. Kumar, B. Kositsup, S. Baruah \& J. Dutta. (2011). Effects of zinc oxide nanoparticles on roots of rice Oryza sativa L. International Conference on Environment and BioScience, 172-176.

4. Chandrasekhar, C., \& U. Bangarusamy. (2003). Maximizing the yield of mung bean by foliar application of growth regulating chemicals and nutrients. Madras Agricultural Journal 90 (1/3), 142-145.

5. C. P. G., T. (2012). Crop Production Guide. Tamil Nadu Agricultural University, Coimbatore, Tamil Nadu.

6. Dass, A., \& S. Sudhishri. (2010). Intercropping in fingermillet (Eleusine coracana) with pulses for enhanced productivity, resource conservation and soil fertility in uplands of Southern Orissa. Indian Journal of Agronomy 55 (2),89-94.

7. Dass, A., S, Sudhishri, \& N. Lenka. (2013). Integrated nutrient management to improve finger millet productivity and soil conditions in hilly region of Eastern India. Journal of crop improvement 27 (5),528-546. https:// doi.org/10.1080/15427528.2013.800828.

8. Divya, G., K. Krishnamurthy \& D. Gowda. (2013). Growth and instability analysis of finger millet crop in Karnataka. Mysore Journal of Agricultural Sciences 47 (1),35-39.

9. Fawusi, M., S. Wanki \& D. Nangju (1982). Plant density effects on growth, yield, leaf area index and light transmission on intercropped maize and Vigna unguiculata (L.) Walp. in Nigeria. The Journal of Agricultural Science 99 (1), 19-23.

10. Genc, Y., G. K. McDonald, \& R. D. Graham (2006). Contribution of different mechanisms to zinc efficiency in bread wheat during early vegetative stage. Plant and soil 281 (1),353-367.

11. Gnanasangeetha, D. \& D. SaralaThambavani (2013). One pot synthesis of zinc oxide nanoparticles via chemical and green method. Research Journal of Mater Science 2320,6055 .

12. Gunasekar, J., A. Kamaraj \& S. Padmavathi (2017). Effect of botanical seed priming on seed quality charactersinblackgram [vignamungo (I.) Hepper] cv. CO6. Plant Archives 17 (2),1383-1387.

13. Jayarambabu, N., B. S. Kumari, K. V. Rao \& Y. Prabhu (2014). Germination and growth characteristics of mungbean seeds (Vigna radiata L.) affected by synthesized zinc oxide nanoparticles. International Journal of Current Engineering and Technology 4 (5),2347-5161.

14. Kaushik, S. \& T. Sharma (2017). Wheat and component intercrop yield, land equivalent ratio and monetary indices influenced by intercropping and row proportions. International Journal of Current Microbiology and Applied Science, 6 (7), 2626-2631. https://doi.org/10.20546/ijcm as.2017.607.370.

15. Koti, R., U. Mummigatti, C. Nawalgatti, F. Savita, M. Guled \& A Anand. (2009). "Complimentary effect of zinc application on iron content in sorghum genotypes. Indian Journal of Plant Physiology, 14 (1),78-81. 
16. Kumar, B. P., S. Halikatti, S. Hiremath \& B. Chittapur (2008). Effect of intercropping system and row proportions on the growth and yield of little millet and Pigeonpea. Karnataka Journal of Agricultural Sciences, 21 (4), 479-481.

17. Manjunath, M., S. Salakinkop \& G. Somanagouda (2018). Productivity and profitability of soybean-based millets intercropping systems. Research on Crops, 19 (1),43-47. http:// dx.doi.org/10.5958/2348-7542.2018.00 006.2

18. Manonmani, V. \& P. Srimathi (2009). Influence of mother crop nutrition on seed yield and quality of blackgram. Madras Agricultural Journal, 96, (1/6),125-128.

19. Mazaherinia, S, A. R. Astaraei, A. Fotovat \& A Monshi. (2010). Nano iron oxide particles efficiency on Fe, Mn, Zn and $\mathrm{Cu}$ concentrations in wheat plant. World Applied Sciences Journal 7.

20. Ministry of Agriculture \& Farmers Welfare Govt. of India (2017). Selected State-wise area, production and productivity of finger millet, Govt. of India. In: https:// www.indiastat.com.

21. Mishra, S. N., A. Chaurasia, V. Tripathi \& B, Kumar (2017). Effects of seed priming methods on growth and Nodulation characters in Pigeonpea (Cajanus cajan L.) seeds. Journal of Pharmacognosy and Phytochemistry, 6 (4),620-623.

22. Mobasser, H. R., M. R. Vazirimehr \& K Rigi (2014). Effect of intercropping on resources use, weed management and forage quality. International Journal of Plant, Animal and Environmental Sciences. 4 (2),706-713.

23. Ozkutlu, F., B. Torun \& I. Cakmak (2006). Effect of zinc humate on growth of soybean and wheat in zinc $\square$ deficient calcareous soil. Communications in Soil Science and Plant Analysis 37 (15-20),2769-2778.

24. Pandey, A. C., S. S. Sanjay, \& R. S. Yadav (2010). Application of $\mathrm{ZnO}$ nanoparticles in influencing the growth rate of Cicer arietinum. Journal of Experimental Nanoscience, 5 (6),488-497.

25. Pawar, V, \& S Laware. (2018). Seed priming a critical review. International Journal of Scientific Research in Biological Sciences 5, 94-101. https:// doi.org/10.26438/ijsrbs/v5i5.94101.

26. Poornima, R, \& R Koti. (2019). Effect of nano zinc oxide on growth, yield and grain zinc content of sorghum (Sorghum bicolor). Journal of Pharmacognosy and Phytochemistry, 8 (4),727-731.

27. Prasad, T., P. Sudhakar, Y. Sreenivasulu, P. Latha, V. Munaswamy, K. R. Reddy, T. Sreeprasad, P. Sajanlal \& T. Pradeep. (2012). Effect of nanoscale zinc oxide particles on the germination, growth and yield of peanut. Journal of plant nutrition 35 (6),905-927.

28. Reddy, D. R., P. S. Pillai, J. John, A. Sajeena \& J. Aswathy (2021). Growth and yield of pulses as influenced by intercropping with finger Millet (Eleusine coracana L. Gaertn.) in the Southern Laterites of Kerala. Legume Research-An International Journal, 1,5.

29. Rehman, A., M. Yasin, M. Akram \& Z. Awan (2002). Response of $\mathrm{Zn}$ applied and $\mathrm{N}$ sources in calcareous soils.
Science Vision 8,100-104.

30. Reynolds, G. H. (2002). Forward to the Future: Nanotechnology and regulatory policy: Pacific Research Institute. Harvard Journal of Law \& Technology. http://surl.li/axwsk.

31. Sadashiv, B., NaN, B. (2004). Production potential of hybrid cotton (Gossypium hirsutum) based vegetable intercropping systems under irrigation. M.Sc. (Agri.) Thesis., University of Agricultural Sciences, Dharwad, India.

32. Sakamma, S., K. Umesh, M. Girish, S. Ravi, M. Satishkumar \& V. Bellundagi. (2018). "Finger millet (Eleusine coracana L. Gaertn.) production system: status, potential, constraints and implications for improving small farmer's welfare. Journal of Agricultural Science (1),162-179. https:// doi.org/10.5539/jas.v10n1p162.

33. Saraswathi, YVS, M. Dinesh Kumar \& K.T. Gurumurthy. (2019). Effect of Nano ZnO on Growth and Yield of Finger Millet [Eleusine coracana (L.) Garten.]. International Journal of Current Microbiology and Applied Sciences, 8,13651371. doi: 10.20546/ijcmas.2019.802.160. https://doi.or g/10.20546/ijcmas.2019.802.160.

34. Sedghi, M., M. Hadi \& S. G. Toluie (2013). Effect of nano zinc oxide on the germination parameters of soybean seeds under drought stress. Annales of West University of Timisoara. Series of Biology, 16 (2),73.

35. Singh, H, R. K. Jassal, J. Kang, S. Sandhu, H. Kang \& K. Grewal. (2015). Seed priming techniques in field crops- A review. Agricultural Reviews, 36 (4), 251-264. http://10.0.7 3.117/ag.v36i4.6662.

36. Slaton, N. A., C. E. Wilson, S. Ntamatungiro, R. J. Norman \& D. L. Boothe (2001). Evaluation of zinc seed treatments for rice. Agronomy Journal ,93 (1),152-157. DOI:10.2134/AGRONJ2001.931152X

37. Stein, A. J., J.. Meenakshi, M. Qaim, P. Nestel, H. Sachdev \& Z. A. Bhutta (2008). "Potential impacts of iron biofortification in India." Social Science \& Medicine 66 (8), 1797-1808.

38. Subramanian, K., \& J. Tarafdar. (2011). Prospects of nanotechnology in Indian farming. Indian Journal of Agricultural Sciences 81 (10), 887-893.

39. Thobatsi, J. T. (2009). Growth and yield responses of maize (Zea mays L.) and cowpea (Vigna unguiculata L.) in an intercropping system. MSc., (Agric), University of Pretoria. http://hdl.handle.net/2263/28657.

40. Tomar, G., S. Taunk \& J. Choudhary. (2011). Book on Science of Crop Production: Part I. Kharif Crops: Kushal Publications and Distributors.

41. Tripathi, A., \& H. Kushwaha (2013). Performance of pearlmillet (Pennisetum glaucum) intercoppped with pigeonpea (Cajanus cajan) under varying fertility levels in the rainfed environment of Bundelkhand region. Annals of Agricultural Research, 34 (1)

42. Yang, Z., J. Chen, R. Dou, X. Gao, C. Mao \& L. Wang (2015). Assessment of the phytotoxicity of metal oxide nanoparticles on two crop plants, maize (Zea mays L.) and rice (Oryza sativa L.). International Journal of Environmental Research and Public Health, 12(12),15100-15109. 